\title{
Development of a Simple Software Program Used for Evaluation of Plasma Electron Density in LIBS Experiments via Spectral Line Shape Analysis
}

\author{
Ashraf Mohmoud El Sherbini ${ }^{1,2}$, Abdel Aziz Saad Al Aamer ${ }^{1}$ \\ ${ }^{1}$ Department of Physics, Collage of Science, Al Imam Muhammad Ibn Saud Islamic University (AMISU), Al Riyadh, KSA; \\ ${ }^{2}$ Laboratory of Lasers and New Materials, Department of Physics, Faculty of Science, Cairo University, Giza, Egypt. \\ Email: elsherbinia@yahoo.com
}

Received August $10^{\text {th }}, 2012$; revised September $14^{\text {th }}, 2012$; accepted September $28^{\text {th }}, 2012$

\begin{abstract}
A Software program has been developed in order to perform a fast and reliable calculation to plasma electron density in laser induced breakdown spectroscopy (LIBS) experiments. This program is based on analyzing the emitted spectral line shape via utilizing facilities of the MatLab $7^{\circledR}$ package to perform this task. This software can perform the following tasks; read the exported data file ( ${ }^{*}$ txt-format) from ICCD camera-software, specify the working wavelength of interest, removes the continuum emission component appeared under the line, calculates the spectral line intensity of the line, calculates the spectral shift of the line from the tabulated values, correct against spectral shift jitter at the peak emission, de-convoluting and extracting the different components contributing to the emitted line full width at half of the maximum (FWHM) and finally calculates the plasma electron density. In this article we shall present the results of the test measurement of the plasma electron density utilizing spectral line shape analysis to the emitted $\mathrm{H}_{\alpha}$-line, Si I-line at $288.15 \mathrm{~nm}$ and $\mathrm{O}$ I-line at $777.2 \mathrm{~nm}$ at different camera delay times ranging from 1 to $5 \mu \mathrm{s}$.
\end{abstract}

Keywords: Software; LIBS; $\mathrm{H}_{\alpha}$-Line; Electron Density; MatLab $7^{\circledR}$

\section{Introduction}

LIBS, is an acronym derived from the letter words of the statement "Laser Induced Breakdown Spectroscopy"[1]. It is a technique based on utilizing light emitted from plasma generated via interaction of a high power lasers with matter (solid, liquid or gases). Assuming that light emitted is sufficiently influenced by the characteristic parameters of the plasma, the analysis of this light yields considerable information about the elemental structure and the basic physical processes in plasma [2]. The thermodynamic state of plasma can be identified via two independent measurable parameters namely; electron density and temperature [2]. In the passive optical emission spectroscopy (OES) mode, light analyzers (spectrographs or monochromators) with a graphical readout are employed to give the characteristic emission spectrum. A spectrum is the functional dependence of the output light spectral intensity (radiance) on the emission wavelength [3-5].

Among the OES methods proposed for the measurement electron density, the spectral broadening of emission lines due to the Stark effect is the most widely use method [3]. This method is based on the assumption that the Stark effect is the dominant broadening mechanism, in comparison to the Doppler and other broadening mechanisms (resonance and Van der Waals broadenings). The validity of this assumption was generally admitted in LIBS experiments and was justified in various studies [6].

Over the tremendous number of the recorded spectra under a variety of different experimental conditions, the extraction of the information's from spectra provides a strong motivation to a fast and precise computer routine for calculation of plasma parameters. This information's are generally stored in the spectral line width (FWHM) and the signal height (spectral radiance). The FWHM is a sensitive function of the Stark and other broadening mechanism, while the spectral radiance is sensitive to plasma temperature and/or elemental concentration [7].

A computer programming used for spectral line shape analysis is not new, several authors have contributed by their work in this field, but the exact processing method were almost not clear which might lead to confusion [8-10]. Several software simulation programs based on different theories were developed to calculate the electron density from the FWHM of the hydrogen lines at the 
Balmer series [11,12].

In this article we will present the results of using a straight forward computer routine written in Matlab ${ }^{\circledR}$ package used for fast and reliable calculation of the plasma electron number density. A spectral line shape analysis was adopted to extract the Lorentzian component of the emitted line FWHM. This software was applied to different emitted spectral lines $\left(\mathrm{H}_{\alpha}\right.$-line, Si I-line at $288.15 \mathrm{~nm}$ and $\mathrm{O}$ I-line at $777.2 \mathrm{~nm}$ ) from plasmas formed via interaction of a high peak power laser with a plane solid target in open air.

\section{Spectral Line Shape Analysis}

\subsection{Shape of Emitted Line}

The emission spectral line shape is the functional relation between the spectral radiance over the wavelength range. This shape describes the distribution of the light around the central emission wavelength. It could be a Gaussian or Lorentzian, depending on the physical effect considered on the emitting atom or ion $[4,5]$.

The Gaussian line shape can be described by [5];

$$
G(\lambda)=A \exp \left(2.7726\left(\frac{\lambda-\lambda_{o}}{\Delta \lambda_{G}}\right)^{2}\right)
$$

With Gaussian half width in (nm) can be expressed as;

$$
\Delta \lambda_{G}(\mathrm{~nm})=7.17 \times 10^{-7} \lambda_{o}(\mathrm{~nm}) \sqrt{\frac{T_{a}(\text { kelvin })}{M_{a}(\mathrm{amu})}}
$$

whereas, $T_{a}$ is the atomic kinetic temperature, $M_{a}$ is the atomic weight in the units of (amu).

These expressions describe the homogeneous distribution of the spectral intensity around the line central wavelength $\left(\lambda_{o}\right)$, with amplitude defined by factor $A$ and $\Delta \lambda_{G}$ is the FWHM. This distribution is best suited to describe Doppler-effect as well as the intrinsic instrumental broadening with $\Delta \lambda_{G}$ is the measured spectrograph bandwidth.

On the other hand, if the radiance across the line shape is not homogeneous, the distribution of the light around the central wavelength can be best described by the Lorentzian function [5]:

$$
L(\lambda)=\frac{0.15915 \Delta \lambda_{s}}{\left(\lambda-\lambda_{o}\right)^{2}+\left(0.5 \Delta \lambda_{s}\right)^{2}}
$$

This FWHM $\left(\Delta \lambda_{s}\right)$ is a direct function of the physical processes that can cause such broadening, e.g. Stark effect and/or pressure broadening.

In plasma spectroscopy the actual emission spectral line is often contains a combination of both Gaussian and Lorentzian shapes. This was attributed to the existence of the different effects of plasma on the measured line shape e.g. Doppler effect, instrumental as well as the Stark effect on the emitting species in the plasma. As a result, the measured line shape should be expressed as the convolution between such effects, which is known as the Voigt line shape [5]:

$$
\begin{aligned}
& V\left(\lambda ; \Delta \lambda_{G}, \Delta \lambda_{S}\right) \\
& =\int_{-\infty}^{\infty} G\left(\lambda^{\prime} ; \Delta \lambda_{G}\right) *{ }^{\prime} L\left(\lambda-\lambda^{\prime} ; \Delta \lambda_{S}\right) \cdot \mathrm{d} \lambda^{\prime}
\end{aligned}
$$

This integration is nothing but the convolution function between Gaussian and Lorentzian function, with a FWHM given by:

$$
\Delta \lambda_{V} \approx \frac{\Delta \lambda_{s}}{2}+\sqrt{\Delta \lambda_{G}^{2}+\left(\frac{\Delta \lambda_{s}}{2}\right)^{2}}
$$

\subsection{Measurement of Plasma Electron Density Utilizing Stark Broadening}

For the hydrogenic lines appeared in normal LIBS experiments, the Stark effect was found as the dominant mechanism of spectral line broadening [7]. The theoretical calculations of Stark broadening of hydrogenic lines parameters were described in detail in several texts [3-6]. For the linear Stark effect, this broadening manifest itself on form of a Lorentzian line shape having a FWHM $\Delta \lambda\left(\mathrm{H}_{\alpha}\right)$, hence, the plasma electron density can be deduced from the spectral broadening of the $\mathrm{H}_{\alpha}$-line utilizing the following expression [13];

$$
n_{e}\left(\mathrm{H}_{\alpha}\right)=8.02 \times 10^{12}\left(\frac{\Delta \lambda_{s}\left(\mathrm{H}_{\alpha}\right)}{\alpha_{1 / 2}}\right)^{3 / 2} \mathrm{~cm}^{-3}
$$

In this expression $\alpha_{1 / 2}$ is the half width of the reduced Stark profiles in $\AA$, it is a weak function of electron density and temperature through the ion-ion correlation and Debye-shielding correction and the velocity dependence of the impact broadening. Precise values of $\alpha_{1 / 2}$ for the Balmer series can be found in Ref. [14].

For an elements other than hydrogen and due to collisions with slow electrons and ions, the quadratic Stark effect acts on the half width at half maximum $\Delta \lambda_{s}$. The electron density can be related to the Lorentzian spectral line half width by [7]:

$$
\Delta \lambda_{s}=[1+1.75 A(1-0.75 R)] \omega_{s} \frac{N_{e}}{N_{e}^{r e f}}
$$

In this equation, $\Delta \lambda_{s}$ is the electron-impact (half) width, $A$ is the ion broadening parameter, which is a measure of the relative importance of the collisions with ions in the broadening, $\omega_{s}$ is the Stark broadening pa- 
rameter. $N_{e}^{\text {ref }}$ is the reference electron density, usually of the order of $10^{16}$ or $10^{17} \mathrm{~cm}^{-3}$, at which the parameters $\omega_{s}$ and $A$ are calculated [7].

It is worth noting that, the Stark broadening of an isolated non-hydrogenic neutral atom spectral line and ion is mainly due to micro-fields produced by the slow electrons. As a consequence, the contribution of quasi-static ions can be neglected and hence Equation (5a) can be approximated to [7];

$$
\Delta \lambda_{s}=\omega_{s}\left(N_{e} / N_{e}^{r e f}\right)
$$

Unfortunately, the situation is not so simple because of the effect of self absorption by non-homogeneous plasma produced by the laser. This is often occur to the emitted spectral lines [15] and might lead to serious errors in the measured density values.

\subsection{The Effect of Self Absorption on Line Shape}

Self absorption acts to distort the line shape. It increases the line width and decreasing the spectral line intensity $[6$, 7]. It is originated mostly from the cooler boundary layer which contains most of population of the neutral atoms [6]. For a strong self absorption the line center may exhibits readily recognizable self-reversal [7].

Not very recently, a new method was developed by the author in order to quantify the effect of self absorption to emitted lines in terms of what is known as the coefficient of self absorption (SA) [16]. This coefficient at the line center $\left(\lambda_{o}\right)$ was originally defined as the ratio of the intensity (counts per sec) of a spectral line subjected to self absorption $I\left(\lambda_{o}\right)$ to that of the same line in the limit of negligible self absorption $I_{o}\left(\lambda_{o}\right)$ and was expressed as [5];

$$
S A=\frac{I\left(\lambda_{o}\right)}{I_{o}\left(\lambda_{o}\right)}=\frac{1-\mathrm{e}^{-k\left(\lambda_{o}\right) \ell}}{k\left(\lambda_{o}\right) \ell}
$$

However, it was suggested that the same amount (SA) can be expressed on the form of relative spectral line widths of the Lorentzian components of the same line in a two quite different situations of self absorbed line $\Delta \lambda_{s \ell}$ to the case of negligible absorption $\Delta \lambda_{s o}$ and therefore, Equation (6a) should reads[16];

$$
S A=\left(\frac{\Delta \lambda_{s \ell}}{\Delta \lambda_{s o}}\right)^{\frac{-1}{0.56}}
$$

Knowing that the Lorentzian FWHM of any spectral line can be expressed in terms of the electron density $\Delta \lambda \approx 2 \omega_{s} n_{e}$, then Equation (6b) can further be modified to express the coefficient of the self absorption in terms of the ratio of two electron density values. One is deduced from the distorted line (which of course will yield larger apparent values) $\Delta \lambda_{s \ell}=2 \omega_{s} n_{e}$ (line) and the other is the density of the electrons in the plasma as measured from an optically thin spectral line (The $\mathrm{H}_{\alpha}$-line in our case) $\Delta \lambda_{s o}=2 \omega_{s} n_{e}\left(H_{\alpha}\right)$. Then Equation (6b) can be modified to:

$$
S A=\left(\frac{\Delta \lambda_{s \ell}}{\Delta \lambda_{s o}}\right)^{\frac{-1}{0.56}}=\left(\frac{n_{e}(\text { line })}{n_{e}\left(\mathrm{H}_{\alpha}\right)}\right)^{\frac{-1}{0.56}}
$$

Equation (6c) indicates that the $S A$ coefficient varies from 1 in case of perfectly optically thin line to the limit of zero in case of completely absorbed line [15].

Finally, one has to utilize Equation (6c) in order to calculate the amount of absorption ( $S A)$, and then use Equation (6a) to get the corrected value of the spectral line intensity $I_{o}\left(\lambda_{o}\right)$.

\section{Software Program}

\subsection{Program Orders to Extract Information from the Recorded Line Shape}

1) Read the spectrum file data (as exported from camera software);

2) Plot the spectrum in a MatLab7 window;

3) Remove the continuum component under the whole of spectrum;

4) Isolate the line of interest (working line);

5) Calculate the wavelength $\left(\lambda_{\text {oexp }}\right)$ at the peak emission;

6) Calculate the spectral shift, from the tabulated wavelength $\lambda_{\text {oTh }}$;

7) Measure the spectral line intensity (Signal height and/or area under curve);

8) Measure the amount of continuum under this line (Background);

9) Calculate the signal to background (continuum) ratio;

10) Building up the necessary functions around this $\lambda_{\text {oexp }}$;

11) Carrying out the necessary convolution between theoretical functions to get the theoretical line shape (Voigt) around this $\lambda_{\text {oexp }}$;

12) Compare the theoretical Voigt function to the isolated measured line shape;

13) Use the necessary equations to calculate the electron density;

14) Repeat the process of comparison until the best fitting is reached, then terminate and give output results;

15) For suspected lines calculate the amount of absorption $(S A)$.

\subsection{Program Flowchart}

As shown in Figure 1, we have started with reading the 


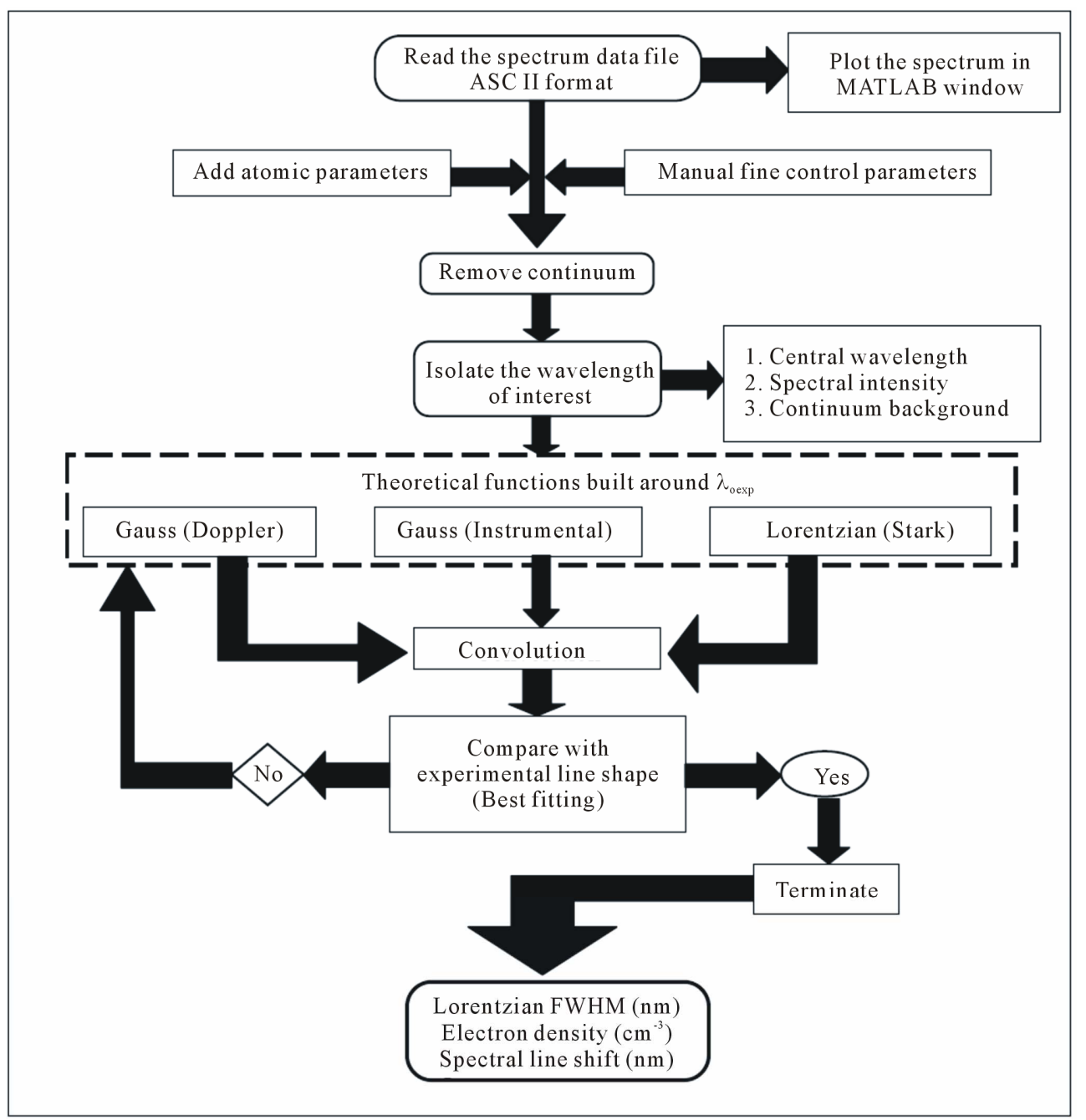

Figure 1. The program flow chart.

exported data from the ICCD camera, and then we feed the atomic data parameters (e.g. wavelength of interest, atomic weight, etc.) and the manual control parameters. Next, we have removed the continuum under the whole the spectrum via creating a new base line. Next we isolate the line of interest, and during this process we calculated the line intensity, and the amount of the continuum appeared under this line and the signal to background ration and also the central line wavelength at the peak of emission. Then, we have built up the required Voigt function via convolution between the Lorentzian and Gauss functions and finally we compare this function to the experimentally measured line shape extracting the Stark component that will be used to measure the electron density at the best fitting. The details of the program can be found at Table 1.

\section{Results and Discussion}

Figure 2 represents the master optical signals in the range from $200-1000 \mathrm{~nm}$ as recorded by the ICCD camera program (KestrelSpec ${ }^{\circledR}$ 3.96) and plotted in a MatLab7 ${ }^{\circledR}$ window. These spectra were taken at a gate time of $1 \mu$ s and arbitrary delay times of 1,3 and $5 \mu$ s.

One can notice the strong continuum component appeared under the whole the spectrum. This continuum is usually attributed to the free-free and free-bound transitions of the quasi free electrons in the plasma and should be removed before processing any optical signal. Also one can notice the decrease in the continuum level as the delay time increases from 1 to $5 \mu \mathrm{s}$.

Figure 3 shows the new base line plotted in red color, this line was created via fitting of the whole the spectrum (40001 pixels) to a polynomial function set at the $5^{\text {th }}$ order. One can notice that for every region the cutting level should be manually adjusted. This can be done by the manual control set at program statement number 15 . The clean spectrum after subtraction of the continuum is shown in Figure 4. 

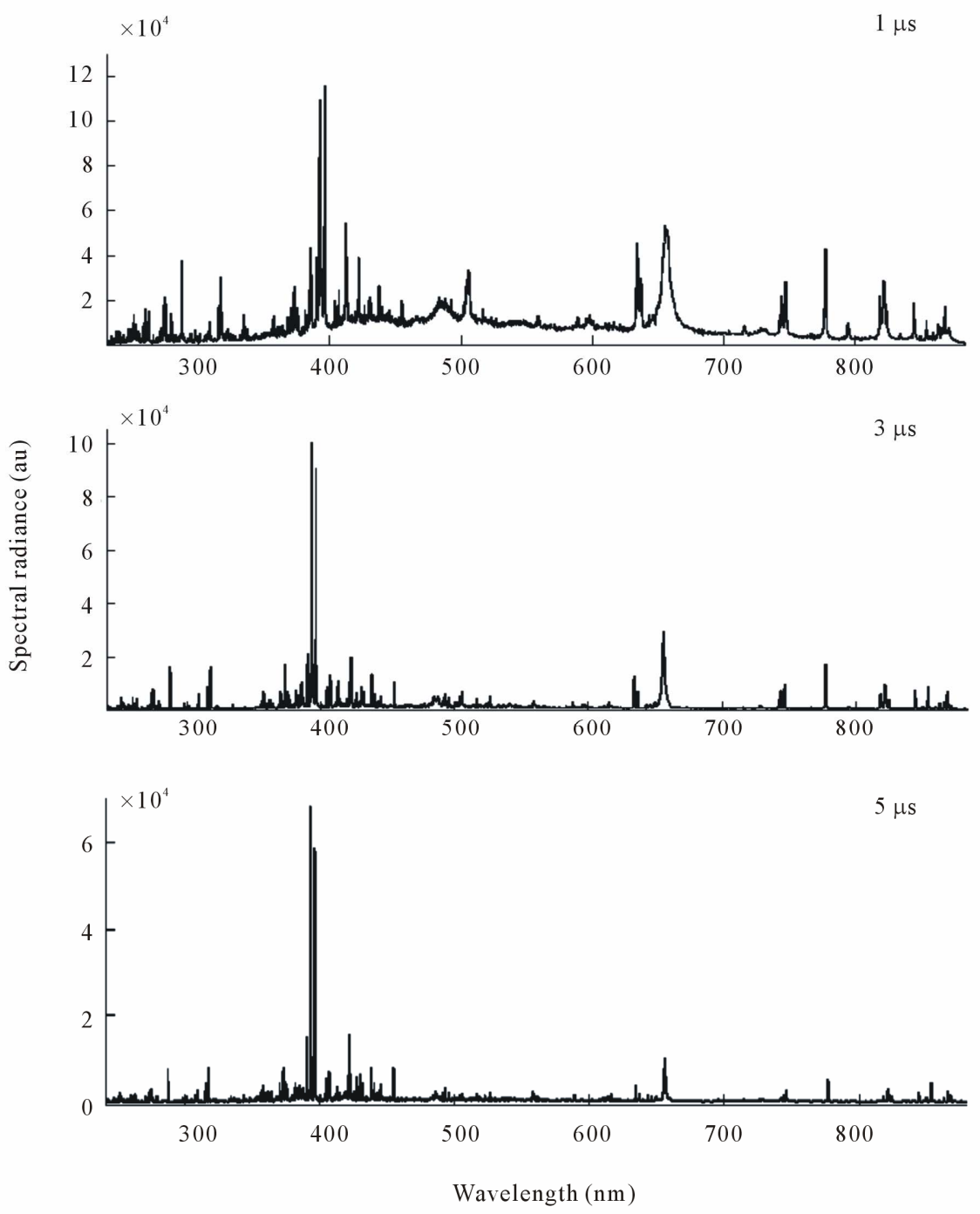

Figure 2. An example of the emitted spectra from plasma at three arbitrary different delay times of 1,3 and $5 \mu$ s.

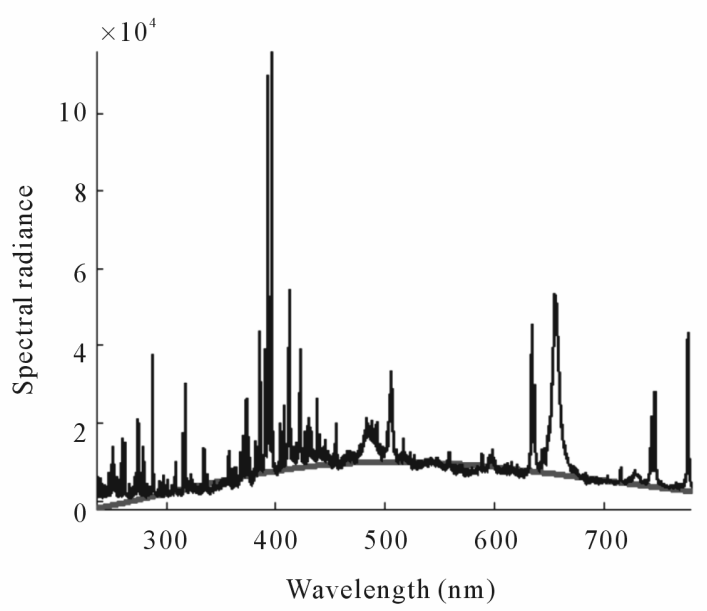

Figure 3. A plot of the master signal (black) and new base line (red).

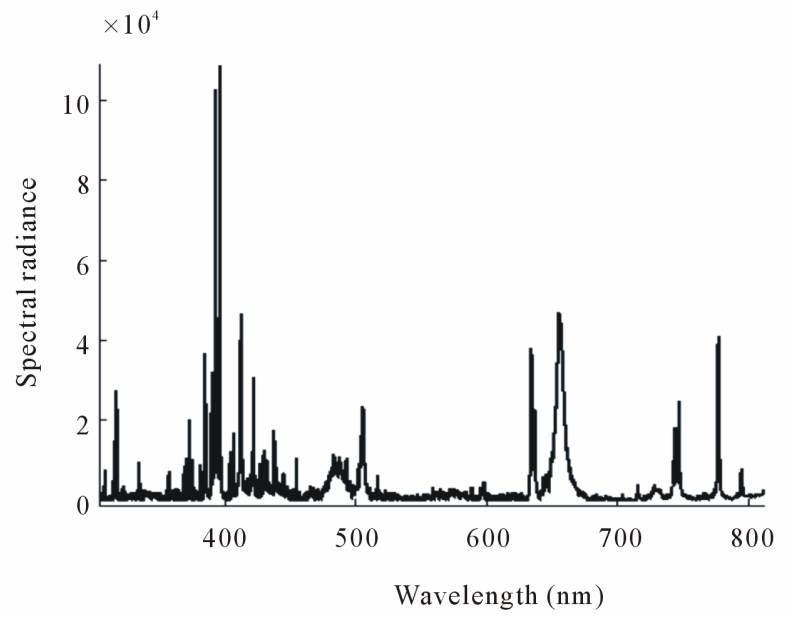

Figure 4. A plot of the emission spectrum after subtraction of the continuum component. 
The isolated $\mathrm{H}_{\alpha}$-line in the master form (data pixel points) is shown at Figure 5. The signal analysis will be applied to this optical signal, first we will calculate the spectral radiance and then de-convolute the different contributions to the line FWHM. Peering in mind that the Stark width component will be used to evaluate the electron density, hence, a comparison of this signal to a theoretically Voigt function will be applied.

On the other hand, Figure 6 shows a plot of the different theoretical functions (Lorentzian and two Gaussian shapes) centered at the experimentally measured central emission wavelength $\left(\lambda_{\text {oexp }}\right)$. It is worth noting that the

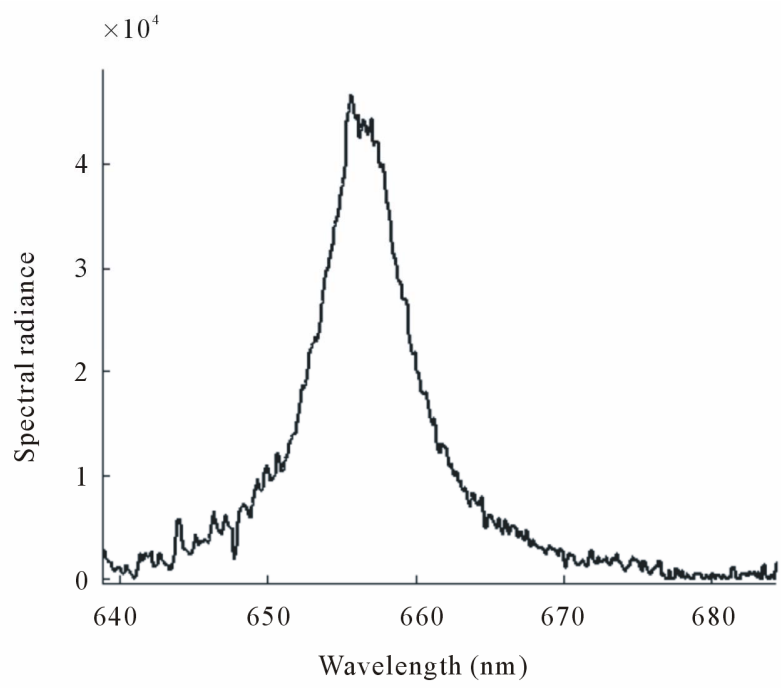

Figure 5. Shown is the isolated master optical emission signal at the $\mathrm{H}_{\alpha}$-line.

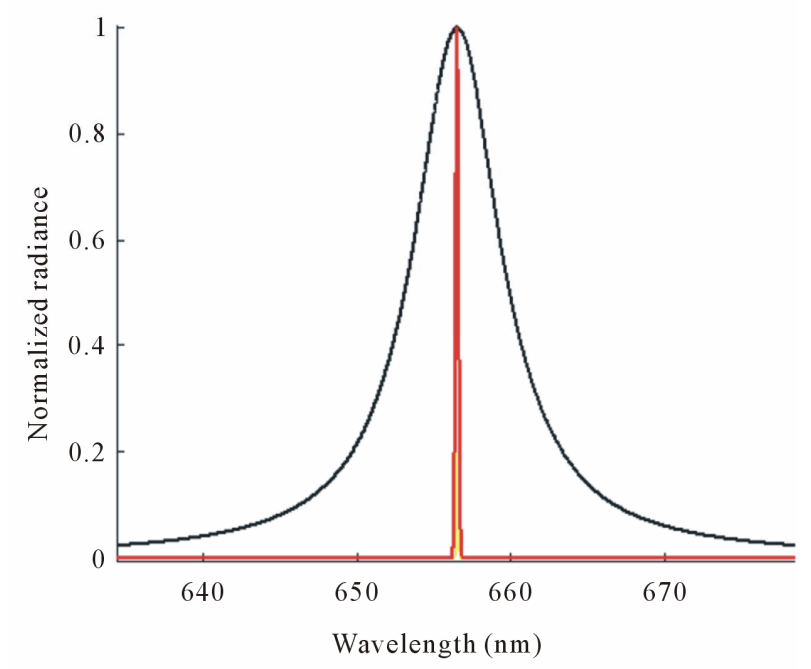

Figure 6. A plot of the Lorentzian function (Drawn in black); the Gaussian function resulted from the instrumental broadening (drawn in red), the other Gaussian resulted from the Doppler-Effect is extremely small.
Gaussian functions can be attributed to the Doppler-effect as well as the instrumental bandwidth of the spectrograph, while the Lorentzian component to the pressure or Stark effect. One can observe that either of the Gaussian components of the line is very small with respect to the Stark component in LIBS experiments, or hence the Doppler component can generally be neglected without too much error. Figure 7 shows the results of the comparison of the theoretical Voigt line shape (Black curve) to the experimentally measured profile (red curve) at the best fitting.

Figure 8 shows the results of fitting of the Voigt function to the $\mathrm{H}_{\alpha}$-line profile at two arbitrary delay times of $3,5 \mu \mathrm{s}$, respectively. The repeated application of the program at two different delay times yields $\Delta \lambda_{\text {Stark }}=1.76$, $1.03 \mathrm{~nm}$, respectively and hence an electron densities of $2.1 \times 10^{17}$ and $9.5 \times 10^{16} \mathrm{~cm}^{-3}$, respectively.

We have tested the program in order to evaluate the electron density from elements other than hydrogen, e.g. Si I at $288.15 \mathrm{~nm}$ and O I at $777.2 \mathrm{~nm}$ appeared in the same emission spectra. Noting that, the equation used to evaluate the electron density should be changed, i.e. instead of using Equation (4), one should use Equation (5b). The stark broadening parameters of the two lines are taken from tables at Ref. [4], at the given reference electron density. At Figure 9 we give the results of the application of the program to the Si I and O I at three different delay times namely at $1,3,5 \mu$ s together with the evaluated electron densities.

Finally, in order to realize the importance of the measurements of the electron densities utilizing different lines arising from plasma produced by laser, one should plot the variation of the electron density as inferred from dif-

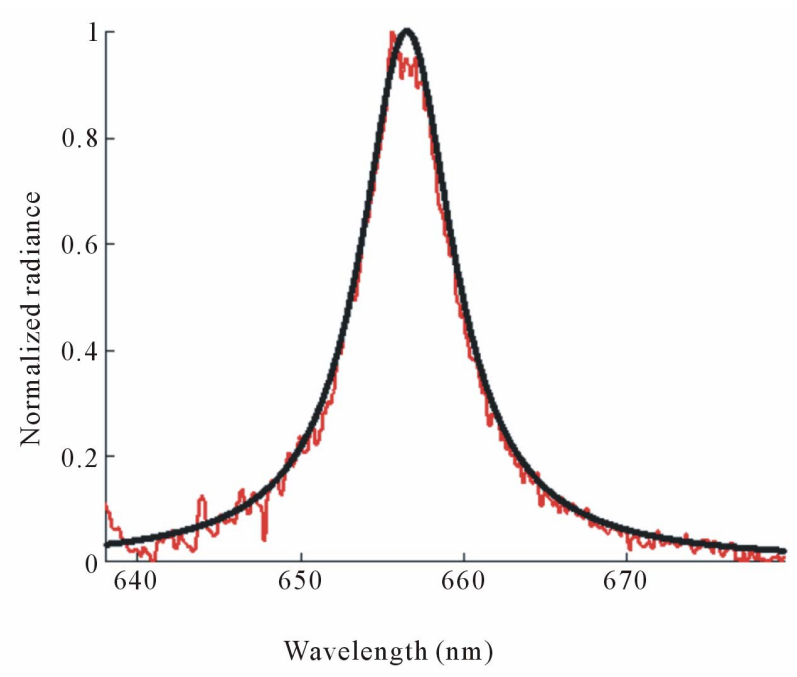

Figure 7. An example of fitting the experimental line profile at the $\mathrm{H}_{\alpha}$-line (red curve) to the theoretical Voigt function (Black curve). 


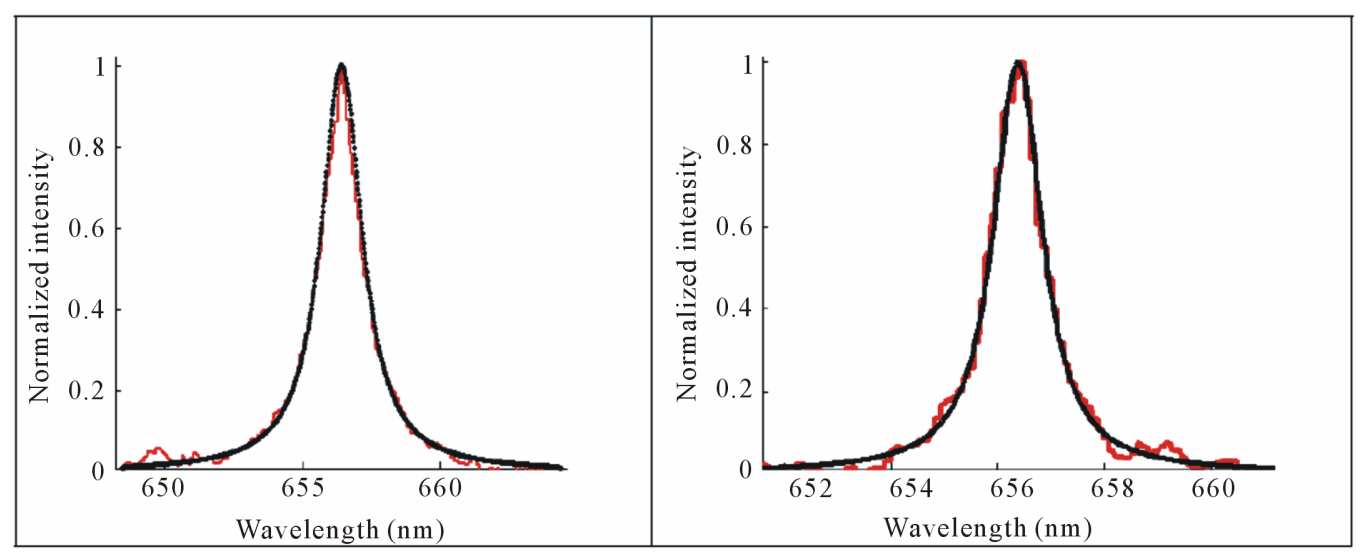

Figure 8. Shown are the results of the fitting of the experimentally measured line profiles of the $\mathbf{H}_{a}$-line to the theoretical Voigt function at an arbitrary delay times of $3,5 \mu$ s (read from left to right) respectively.

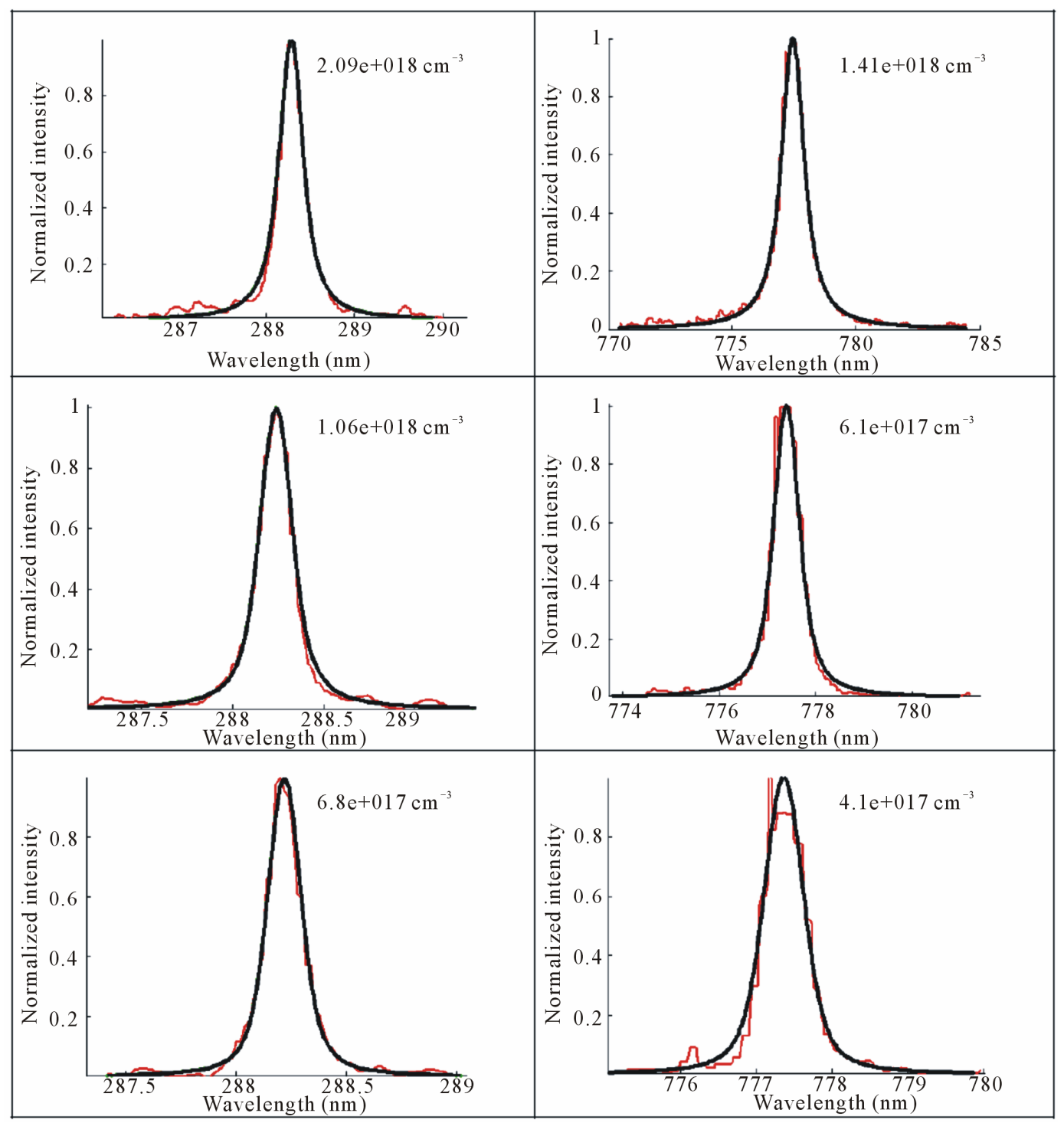

Figure 9. Shown are some examples of the Voigt fitting to the Si I line at $288.15 \mathrm{~nm}$ and the $\mathrm{O}$ I at $777.2 \mathrm{~nm}$, at three different delay times of $1,3,5 \mu$ s (read from top to bottom). 
ferent lines. Figure 10 represents the results of the estimated electron density with delay time. From the figure one can conclude that the line at Si I suffering from optical thickness while this is not the case of the O I at 777.2 $\mathrm{nm}$. This is because one can notice the strong deviation of the electron density calculated from the Si I-line at $288.14 \mathrm{~nm}$ and the moderate deviation of the $\mathrm{O}$ I-line at $777.2 \mathrm{~nm}$ from the density as calculated from the $\mathrm{H}_{\alpha}$-line. This indicates that both lines are subjected to some self absorption.

With the help of expression (6c), after comparison of the calculate electron density from one line to the same amount as evaluated utilizing the $\mathrm{H}_{\alpha}$-line one can calculate the amount of optical thickness of the plasma at each line and correct the intensities of either line which lies after the scope of this article.

\section{Conclusion}

We have developed simple routine software used to evaluate the electron density utilizing the spectral line shape analysis to the light emitted from plasma. This routine is based on comparison of the measured line shape to a theoretically built Voigt function. This enables us to extract the stark component contribution to the line width, hence the calculation of the plasma electron density. The electron density was evaluated utilizing different spectral lines emitted from the plasma under the same condition e.g. the $\mathrm{H}_{\alpha}$-line (that was used as a reference line in the measurement of the electron density) as well as $\mathrm{Si}$ I-line at $288.15 \mathrm{~nm}$ and the O I-line at the $777.2 \mathrm{~nm}$.

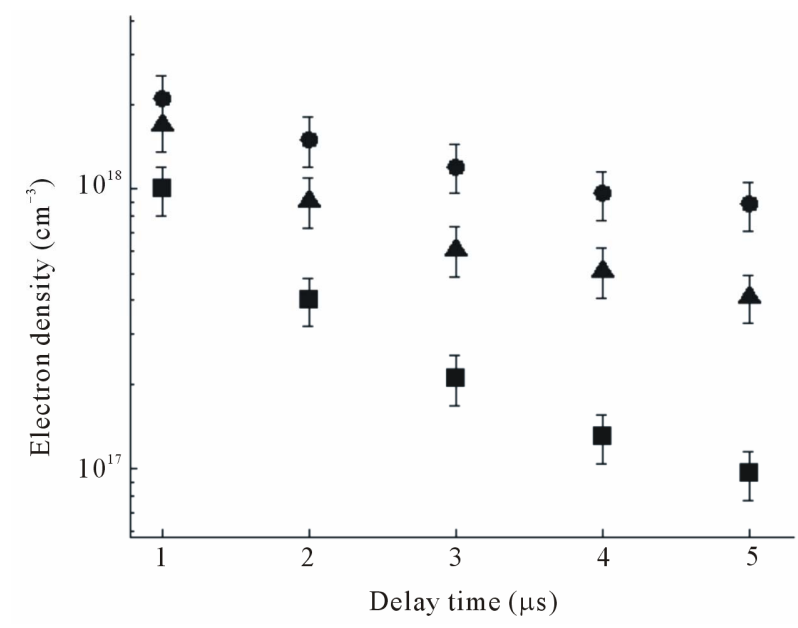

Figure 10. A demonstration to the temporal variation of the finally calculated electron density utilizing the program with delay time, squares represent the calculated electron density from the $\mathrm{H}_{\alpha}$-line, triangles are the same density as measured from the $O \mathrm{I}$ line at $\mathbf{7 7 7 . 2} \mathbf{~ n m}$ and the solid disks are the electron densities as calculated from the Si I-line at $288.15 \mathrm{~nm}$.
The higher densities showed by lines other than hydrogen was attributed to the presence of optical depth of the plasma to these lines.

\section{Notations}

1) In order to change the wavelength of interest (i.e. to apply the analysis to another line) one has to assign the new wavelength of interest at line number 4 .

2) For lines other than the $\mathrm{H}_{\alpha}$-line, one has to use Equation 5(b) instead of Equation (4) and use line number 33 instead of line number 32 (pause line 32 by using the $\%$ sign at the start of the line) together with assignment of the reference electron density $\left(\boldsymbol{N}_{\boldsymbol{r}}\right)$ and Stark broadening parameter $\left(\omega_{s}\right)$ at lines 11,12 .

3) From our experience to handle the LIBS spectra under our condition of the laser energy and duration the expected temperature is almost around $1 \mathrm{eV}$ and hence the Doppler FWHM is almost centered on $0.04 \mathrm{~nm}$ that can be neglected.

4) One more option that can enhance precision of the fitting is to change the value of the controllable precision parameter at line 16 .

5) In order to examine somewhat line against the effect of absorption, one has to run the program two times in series. The first run to calculate the density utilizing the $\mathrm{H}_{\alpha}$-line, and the next to run the program to calculate the same density using the suspected line. Then the application of Equation (6c) will evaluate directly the amount of self absorption. If the SA value is found in the range (0.8 1) the line is considered as optically thin [15], lower values of $S A$ means that the lines is thick and need to be corrected.

6) The correction of spectral intensity against the effect of self absorption can be carried using Equation (6a) in order to evaluate $I_{o}\left(\lambda_{o}\right)$.

7) Lines (13 - 16) completely enables one to control the fitting to the experimental line shape.

\section{Acknowledgements}

The experimental part utilized in the analysis was carried at the Laboratory of lasers and New Materials, Faculty of Science at Cairo University-Egypt under the supervision of Prof. Th. M. EL Sherbini and Prof. S. H. Allam.

\section{REFERENCES}

[1] A. F. M. Y. Haider and Z. H. Khan, "Determination of Ca Content of Coral Skeleton by Analyte Additive Method Using the LIBS Technique," Optics \& Laser Technology, Vol. 44, No. 6, 2012, pp. 1654-1659. doi:10.1016/j.optlastec.2012.01.032

[2] W. Lochte-Holtgreven, "Plasma Diagnostics," North Holland, Amsterdam, 1968. 
[3] H. R. Griem, "Plasma Spectroscopy," McGrow-Hill, Inc., New York, 1964.

[4] H. R. Griem, "Spectral Line Broadening by Plasmas," Academic Press, New York, 1974.

[5] H. J-Kunze, "Introduction to Plasma Spectroscopy," Springer Series on Atomic, Optical and Plasma Physics, Vol. 56, Springer, New York, 2009.

[6] H. Amamou, A. Bois, B. Ferhat, R. Redon, B. Rossetto and P. Matheron, "Correction of the Self-Absorption for Reversed Spectral Lines: Application to Two Resonance Lines of Neutral Aluminum," JQSRT, Vol. 77, No. 4, 2003, pp. 365-372. doi:10.1016/S0022-4073(02)00163-2

[7] N. Konjevic, "Plasma Broadening and Shifting of NonHydrogenic Spectral Lines: Present Status and Applications," Physics Reports, Vol. 316, No. 6, 1999, pp. 339401. doi:10.1016/S0370-1573(98)00132-X

[8] R. Žikić, M. A. Gigosos, M. Ivković, M. Á. González and N. Konjević, "A Program for the Evaluation of Electron Number Density from Experimental Hydrogen Balmer Beta Line Profiles," Spectrochimica Acta Part B, Vol. 57, No. 5, 2002, pp. 987-998. doi:10.1016/S0584-8547(02)00015-0

[9] N. Konjević, M. Ivković and N. Sakan, "Hydrogen Balmer Lines for Low Electron Number Density Plasma Diagnostics," Spectrochimica Acta Part B, Vol. 76, 2012, pp. 16-26.

[10] C. Yubero, M. C. García and M. D. Calzada, "On the Use of the $\mathrm{H} \alpha$ Spectral Line to Determine the Electron Density in a Microwave $(2.45 \mathrm{GHz})$ Plasma Torch at Atmospheric Pressure," Spectrochimica Acta Part B, Vol. 61, No. 5, 2006, pp. 540-544.

\section{doi:10.1016/j.sab.2006.03.011}

[11] W. Olchawa, "Computer Simulations of Hydrogen Spectral Line Shapes in Dense Plasmas," JQSRT, Vol. 74, No. 4, 2002, pp. 417-429. doi:10.1016/S0022-4073(01)00262-X

[12] S.-K. Chan and A. Montaser, "Determination of Electron Number Density via Stark Broadening with an Improved Algorithm," Spectrochimica Acta Part B, Vol. 448, No. 2, 1989, pp. 175-184.

[13] A. M. El Sherbini, H. Hegazy and Th. M. El Sherbini, "Measurement of Electron Density Utilizing the $\mathrm{H}_{\alpha}$-Line from Laser Produced Plasma in Air," Spectrochimica Acta Part B, Vol. 61, No. 5, 2006, pp. 532-539. doi:10.1016/j.sab.2006.03.014

[14] P. Kepple and H. R. Griem, "Improved Stark Profile Calculations for the Hydrogen Lines $\mathrm{H} \alpha, \mathrm{H} \beta, \mathrm{H} \gamma$, and $\mathrm{H} \delta$," Physical Review, Vol. 173, No. 1, 1968, pp. 317-325. doi:10.1103/PhysRev.173.317

[15] A. M. EL Sherbini, A. M. Aboulfotouh, S. H. Allam and Th. M. EL Sherbini, "Diode Laser Absorption Measurements at the $\mathrm{H}_{\alpha}$-Transition in Laser Induced Plasmas on Different Targets," Spectrochimica Acta Part B, Vol. 65, No. 12, 2010, pp. 1041-1046. doi:10.1016/j.sab.2010.11.004

[16] A. M. El Sherbini, Th. M. El Sherbini, H. Hegazy, G. Cristoforetti, S. Legnaioli, V. Palleschi, L. Pardini, A. Salvetti and E. Tognoni, "Evaluation of Self-Absorption Coefficients of Aluminum Emission Lines in Laser-Induced Breakdown Spectroscopy Measurements," Spectrochimica Acta Part B, Vol. 60, No. 12, 2005, pp. 1573 1579. doi:10.1016/j.sab.2005.10.011 


\section{Appendix A}

Program statements in MatLab $7^{\circledR}$.

Table 1. Description to order at each line.

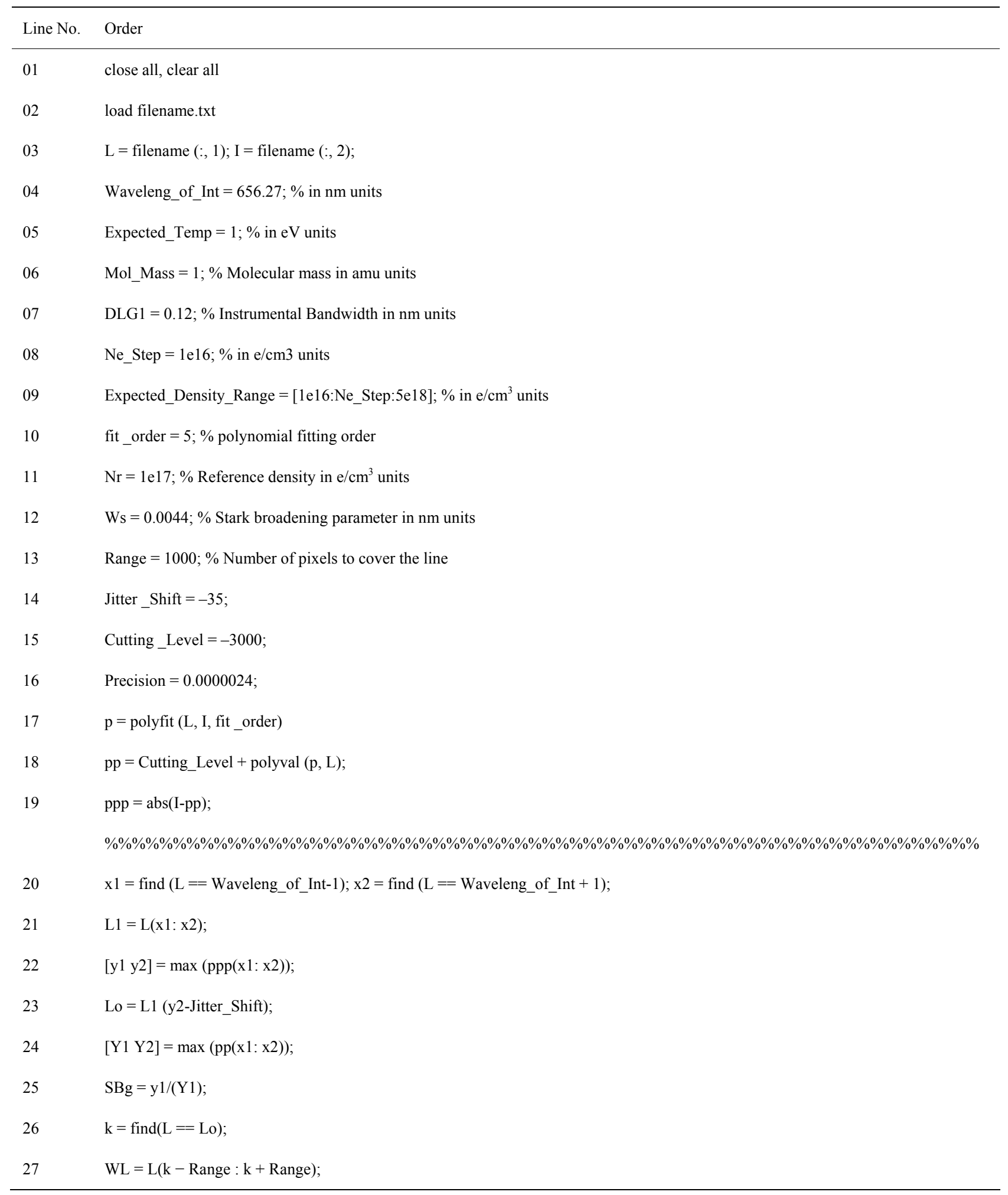




\section{Continued}

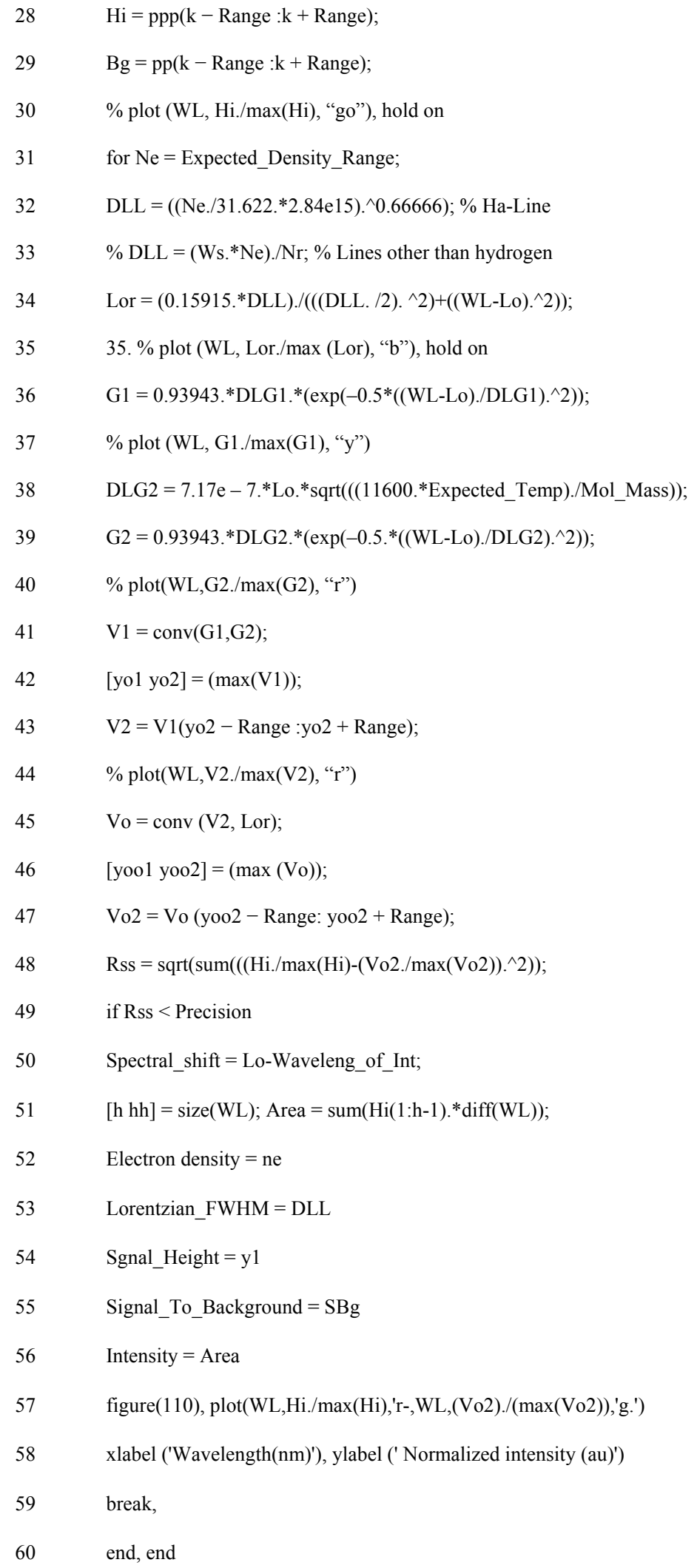




\section{Appendix B}

\section{Program Statement Function}

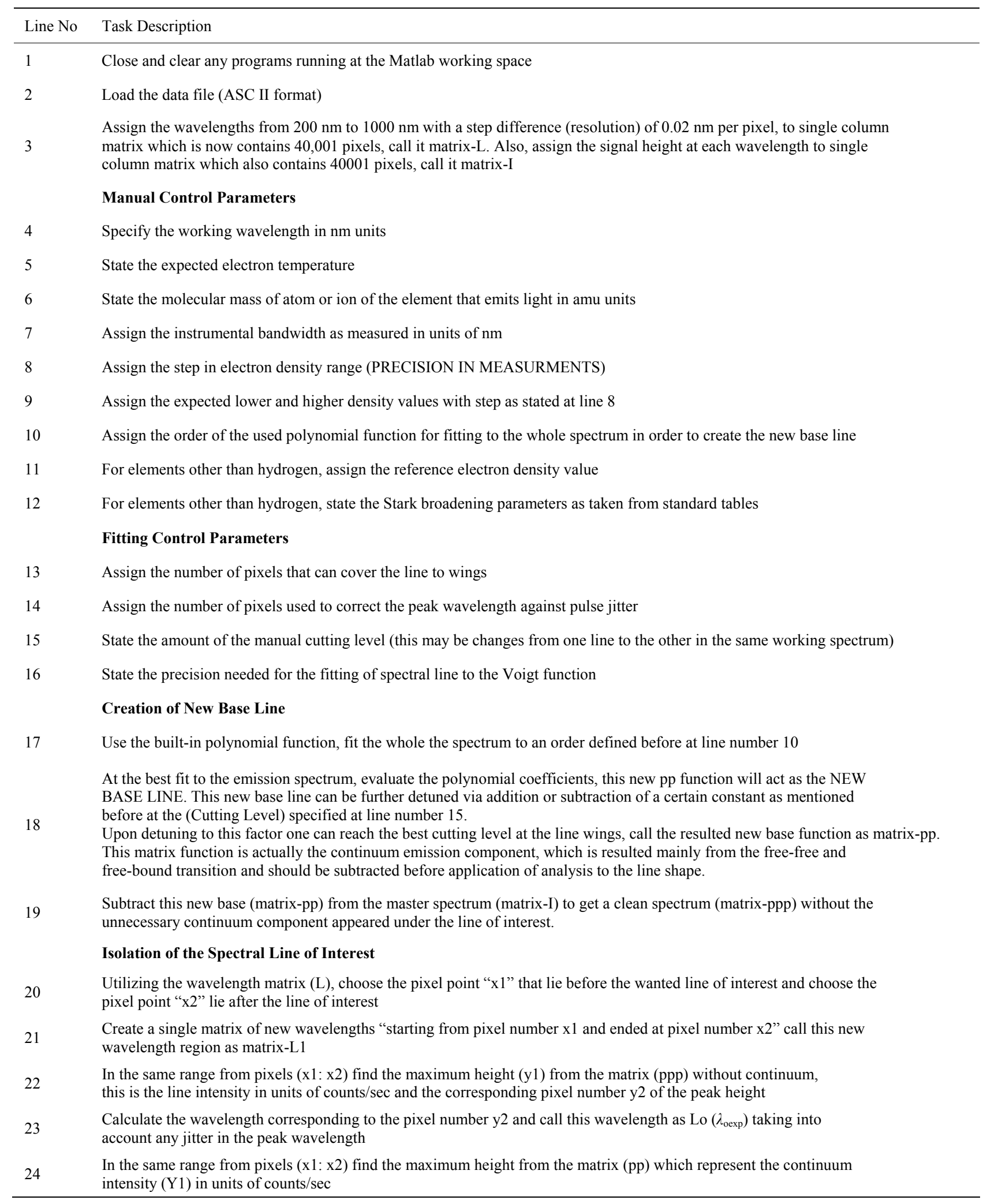




\section{Continued}

25 Calculate the signal to background (y1/Y1)

\section{Built up the Necessary Theoretical Functions around $\lambda_{\text {oexp }}$}

From the wavelength matrix $\mathrm{L}$, find the pixel number (k) corresponding to $\lambda_{\text {oexp }}$

Create a new wavelength region centered around $\lambda_{\text {oxp }}$ and extended by number of pixels specified previously by (Range — at line number 13)

Utilizing matrix (ppp) of signal heights and free from continuum, find the new heights in the same range corresponding to the new wavelength WL regime i.e. in the same region defined by statement range at line 13

Utilizing matrix (pp) of background continuum, fine the new heights in the same range corresponding to the new wavelength WL regime i.e. in the same region defined by statement range at line 13

Plot, if necessary, the experimental isolated line in the new range defined by new wavelength regime WL and the corresponding signal heights $\mathrm{Hi}$

\section{The Theoretical Functions 1. Lorentzian Function}

With the help of the properties of the "FOR-loop" let the electron density $\mathrm{Ne}$ is the free running parameter according to values previously defined at line number 9 , together with the precision step at line 8

For the hydrogen $\mathrm{H}_{\alpha}$-line only use equation 4 at the text to calculate the Lorentzian FWHM in $\mathrm{nm}$

For lines other than the hydrogen $\mathrm{H}_{\alpha}$-line, use this equation $5 \mathrm{~b}$ to calculate the Lorentzian FWHM in nm

Calculate the Lorentzian function according to line FWHM defined in the previous step

Plot, if necessary the theoretical Lorentzian function centered around $\lambda_{\text {oexp }}$ in the new range defined by wavelengths WL and signal heights $\mathrm{Hi}$

\section{Gauss Function Resulted from the Instrumental Bandwidth}

Calculate the Gauss function according to line FWHM defined line number 7

Plot, if necessary the theoretical Gauss function centered around $\lambda_{\text {oexp }}$ in the new range defined by wavelengths WL and signal heights $\mathrm{Hi}$

\section{Gauss Function Resulted from the Doppler Effect}

Calculate the line FWHM according to Doppler effect centered around $\lambda_{\text {oexp }}$ in the new range defined by wavelengths WL and signal heights Hi at the expected electron temperature specified at line number 5

Calculate the Gauss function according to line FWHM defined in the previous step

Plot, if necessary the theoretical Gauss function centered around $\lambda_{\text {oexp }}$ in the new range defined by wavelengths WL and signal heights $\mathrm{Hi}$

\section{Start Convolution between the Different Functions}

Start with convolution between the two Gauss functions G1, G2

Calculate the pixel number corresponding to the maximum of the new function V1 and call this number as yo2

From this function V1 extract the required number of pixels just to cover the spectral line according to Range specified to line 13 , call this function as V2

Plot, if necessary the theoretical V2 function centered around $\lambda_{\text {oexp }}$ in the new range defined by wavelengths WL and signal heights $\mathrm{Hi}$

\section{Carry a Convolution between V2 and the Lorentzian Function}

Convolute the two functions V2 and Lorentzian function (Lor), call new function as Vo

Calculate the pixel number corresponding to the maximum of the new function V1 and call this number as yoo2

From this function Vo extract the required number of pixels just to cover the spectral line according to Range specified to line 13 , call this function as $\mathrm{Vo} 2$

Compare between the theoretically built normalized Voigt function (Vo20 and the experimentally normalized measured line profile $\mathrm{Hi}$ according to the principle of least square fitting method. Call the residual number as RSS

\section{Utilizing the Conditional (IF-Statement)}

If, the residual number at line 48 corresponding to the fitting, is lower than a certain level defined by order precision at line 16 


\section{Continued}

50 Calculate the spectral shift

51 Evaluate the area under the experimentally measured profile

52 Evaluate the electron density at the best fit

53 Evaluate the Lorentzian FWHM in nm at the best fit

54 Evaluate the signal height at the best fit

55 Evaluate the background at the best fit

56 Evaluate the spectral intensity as area under curve at the best fit

56 Plot the final results of comparison between the theoretical function and the experimentally measured line profile

58 Break the loop

59 End the first loop started at line 49

60 End second loop started at line 31 